\title{
A FURTHER SERIES OF ENUCLEATIONS OF THE PROSTATE.
}

\author{
By SIR WILLIAM THOMSON, C.B., F.R.C.S.; \\ Surgeon to the Richmond Hospital; \\ Hon. Surgeon to the King.
}

[Read in the Section of Surgery, May 11, 1906.]

In a paper read before this Section of the Academy and in communications to the journals I have already written on the removal of the enlarged prostate by the suprapubic route. I have dealt with the question whether the high operation or the perineal is the more generally applicable, and which is the safer and the quicker. These are very important considerations when we remember that the majority of our patients are of advanced age, broken down by the insufferable distresses and the pathological mischiefs of the affection; and after some experience I may state my position. I adhere to the view which I originally expressed - that the suprapubic method is in most cases the best. I am aware, of course, that this opinion is not universally held, but I think I may assert confidently that the great majority of those surgeons who have operatively treated enlarged prostate have pronounced in favour of the suprapubic procedure.

To-night I do not intend to raise any serious controversial topic. Who was the originator, whether the gland is removed with its capsule, or without it, are not matters with which I shall venture to trouble you. But I wish to record some additional cases in my own practice, and to refer to a few points which have interested me. The operation has sprung into much prominence, and has been 
widely practised, but as in all cases of new methods there is a good deal to be learned and reported before we arrive at the high level of great comparative safety.

Results.-I submit a tabular list of the cases of enucleation which I have so far done, and the structures which I have removed. They number eighteen in all. The list

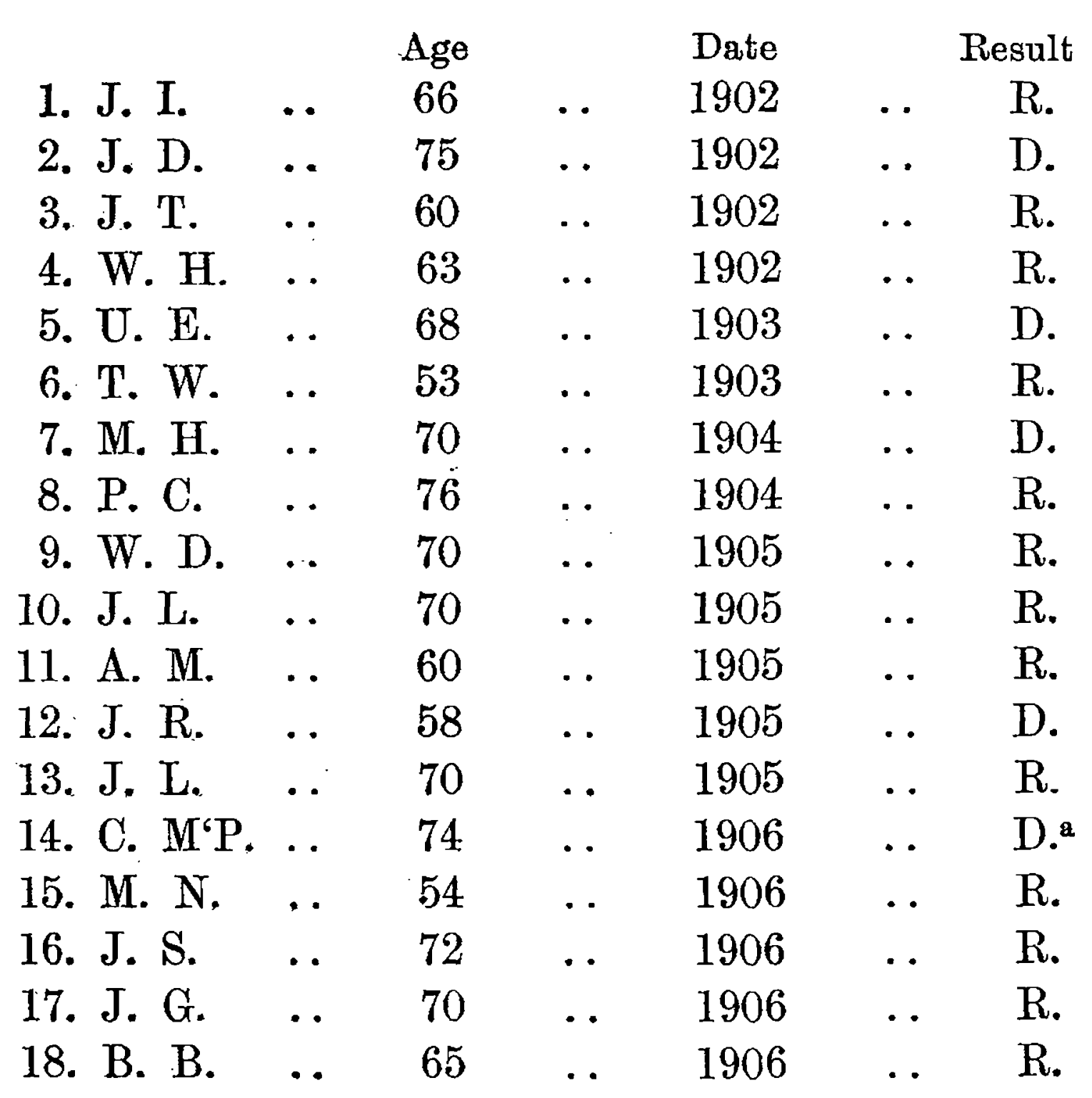

does not include cases in which $\mathrm{I}$ have explored the bladder by the superior incision or established a fistula when the patient's condition was so bad or the age so advanced that it would have been a fatal procedure to carry out enucleation, or cases of malignant disease; nor do I include a case which $I$ did two months ago, in which all the symptoms of prostatic obstruction were present, but with no evidence of enlargement in the rectum. It was one of the rare examples of prostatism without enlargement, but it

" Twelve weeks later, of gangrene of lung. 
does not come within the scope of the present communication. Nine patients were over seventy years of age; the youngest was fifty-four and the oldest seventy-six. Two of the tumours were of very large size- $6 \frac{1}{4}$ ounces, and another 10 ounces less a few grains. Three came away in one mass, including the prostatic urethra. The immediate and remote results did not differ from those following enucleation of the lobes separately. The patients have had no difficulty in emptying the bladder, there has been no irritation or symptom of stricture at the torn vesical extremity of the urethra, and the function of easy urination has been completely restored.

Phosphates.-One of the troubles which sometimes are associated with the operation is the deposit of phosphates on the edges of the wound. I have had several instances of this. Confined to the edges one expects that these deposits will clear away in about ten days, but they are not always limited in this way. In Case IV. this occurred. The encrustation was very extensive and persistent. The patient had so much distress after some months that I was compelled to open the bladder again. I found the mucous membrane coated with phosphates, and there were two phosphatic calculi. These were removed and the deposit was dealt with. There was subsequent copious irrigation, and suitable internal treatment, but when he went home a good deal of irritation still remained, and there had been a recurrence of phosphates. This is the only case in which there has been any persistent phosphatic deposit.

When the prostate has been removed the covering of it which remains in the bladder is very thin. It has been in some parts subjected to much bruising, and its vitality is distinctly lowered. Some of this occasionally dies, and upon it deposits of phosphates may occur. In two cases this happened, and the fragments, finally detached, were 
264 A Further Series of Enucleations of the Prostate.

extruded through the wound. This offers another reason why the opening into the bladder should be shaped by a large tube, about three-quarters of an inch in diameter, for it affords ample space for free washing out and the carrying away of débris and deposit.

In my last ten cases this condition has occurred only three times. In the instances in which there has been no appearance of it I have noticed that there has not been any preceding chronic cystitis, the patients have responded to preliminary treatment satisfactorily, and the bladder has been brought into a fairly healthy state by rest, the careful use of the catheter, washing out, and the administration of some drug to secure acidity of urine. When the symptoms allow of such a course I regard it as of the utmost importance. I have tried the acid phosphate of soda and several other drugs in the post operative course of the case, but I think on the whole I have had the most satisfactory results from benzoate of soda in ten-grain doses, with a drachm of tinct. of buchu, three or four times a day.

Flushing.-But with this must be associated copious flushing of the bladder with boric solution. I have pointed out the importance of a large bladder opening. For some time I have rarely used the catheter for washing out. I suspected that it sometimes did mischief, and so I adopted the plan of first flushing through the upper wound... But this alone is apt to be deceptive. If you pass a stream directly through the bladder wound you will soon find that the fluid is returning perfectly clear. But this current does not effectively stir up the deposit which is lying about the base of the bladder and the original site of the prostate. When I have finished this stage of the washing I pass into the urethra a glass nozzle, about three or four inches long. It should have as wide a mouth as 
it will carry. Attached to a syringe or an irrigator it conveys the fluid in a full stream into the bladder. The elots or other deposits of pus and phosphates are displaced, mixed with the current, and discharged through the suprapubic opening. You will be often surprised to find how much dirt you will get out of a bladder which you may regard as perfectly clean if you confine yourself to the single route washing.

Sloughing.-A more serious danger is the existence of extensive sloughing in the wound and the infection of the layers of cellular tissue in continuation with it. This is especially apt to occur in patients who have large and pendulous abdomens. I lost Case VII. by infection of the deeper parts of the space of Retzius. Sloughing occurred, and it was only towards the end of the third week that the patient showed urgent symptoms. Then $I$ discovered an abscess which I opened above Poupart's ligament, but he died on the twenty-third day, although $I$ had regarded him as quite safe a week before.

A fortnight ago I operated on a gentleman (Case XVIII.) of this type, assisted by Mr. Tobin, Mr. H. Rutherford, and Dr. Boyd.

He had long been troubled with urinary difficulties; but the week before, for the first time, he had complete retention. $\mathrm{He}$ was seen by Dr. Gorham, of Clifden, and Dr. Loftus, of Roundstone, but they failed to get in a catheter, and Dr. Gorham very skillfully aspirated above the pubis. As the obstruction could not be overcome, the operation was repeated next day, and the patient was at once sent on to Dublin - a distance of 160 miles. I saw him the same evening, and, after some difficulty, succeeded in reaching his bladder. He was in a very low state, and there was cystitis. The prostate was so large that my finger in the rectum could not reach its upper limit. I treated him for a week, and operated on 26th of April. The wound passed through nearly three inches of fat, the bladder was quickly reached 


\section{A Further Series of Enucleations of the Prostate.}

and opened, and I then found a prostate of enormous dimensions almost filling the cavity. The right lobe was huge, as I show you, and came away in three portions. The left is much smaller, and was removed in one piece. The total weight is ten ounces, less a few grains, and is the largest $I$ have removed. The enucleation lasted 25 minutes. The upper portions were quickly freed, but the attachments at the base were very close, and required, finally, the use of the two first fingers before the gland could be delivered. Now, in this case I had to deal with a great deal of superficial fat, and a large wound in the abdominal wall was necessary. When my fingers were in the bladder my hand had disappeared in the gap of the surface wound. Much force was necessary, and the operation was most tiring to the hand. In upper part the edges of the wound have united perfectly. On the third day, however, signs of extensive sloughing appeared. The process passed under the abdominal walls on either side for a couple of inches, and the edges lost colour and necrosed. This was sufficient to cause much anxiety.

This untoward complication is especially apt to occur in fat subjects. The tissue is itself of low vitality, and in burying the hand in the wound bruising of the parts is likely to accelerate necrosis.

What I did was to remove a couple of stitches to enable me to expose the deep parts, and then these were bathed in 30 per cent. solution of peroxide of hydrogen. This has been done at each dressing. The temperature has remained normal, the sloughs are separating, the line of demarcation is well established in the skin, and the patient is, I am glad to say, progressing rapidly to recovery.

Hamorrhage from the Prostate.-Case XI. was one of much interest because of the diversity of the views which from time to time were held regarding it. It was diagnosed as stone in the kidneys and malignant disease of the bladder. My own independent declaration was that the case was one of enlarged prostate, with possibly a stone in the bladder. I have already published the full 
details of the case in the British Medical Journal of Jan. 27, 1906, but I may here allude to one or two points of great interest:-

The patient was examined in London, and no stones were found. In two days he had retention for the first time, having never previously needed the use of a catheter. He was sent home, and I found him suffering from bad cystitis. In a fortnight he had a bad attack of hæmorrhage, and in spite of all I could do, by means of styptics internally and locally, the bleeding continued. He was greatly weakened, and, having regard to his alarming condition, I suggested that the bladder should be opened and the prostate removed. In this view the gentlemen who helped me in consultation concurred, and the operation was duly performed. The hæmorrhage immediately ceased. I found no less than 32 calculi, which I show you. The prostate weighed 2 ounces. The patient is now perfectly well, and has never had the least return of his former symptoms, although he suffered for a time from a painful spot in the scar which had to be excised.

The failure by an experienced surgeon to find the stones is only another instance of the difficulty that sometimes impedes us in diagnosis. The important matter, however, is the carrying out of a treatment for the hæmorrhage, which, so far as I know, had not before been reported, and I submit it to the Section as an advance in the treatment of such cases which may be established. If we come to the conclusion that the bleeding is from the prostate, and if the discharge is continuous and excessive, not yielding to any other known remedy, I regard the proceeding as urgently called for. In these cases the covering of the gland is often exceedingly vascular, and the veins are enlarged. If from ulceration or other cause a large continuous hæmorrhage is established, particularly from an open vessel, it does not seem reasonable to hope that it will be stopped by styptics. We cannot apply pressure, and we cannot hope that a clot will help us out of the danger, 
for the temporary covering is certain to be disturbèd by the action of the bladder in its efforts to get rid of an offending body. Urine is constantly distilling into the cavity, and helps to increase the expulsive efforts of the patient, so that the essential condition of rest for the permanent healing of the vessel is absent. What, then, can we do in such a case? To my mind there is no other way open to us but to expose the seat of bleeding, to enucleate the prostate if that be necessary, or to plug the bladder.

An Abdominal Tumour.-Case XV. was sent to me as a tumour of the abdomen. It had been seen by several medical men.

The swelling was very easily observed, occupying the middle of the abdomen and extending above the umbilicus. The patient was able to pass a normal amount of urine in a fair stream, but he was disturbed two or three times at night. He felt no pain, and was able to hold water for four or five hours without distress. The tumour was hard, but it did not give the feeling of solidity. I found that he had an enlarged prostate, and on passing a catheter I drew off 58 ounces of urine. This reaccumulated rapidly, and the next trial produced three pints It was a case of atony due to over-distention, and after a few days I removed his prostate, and in five weeks he went home perfectly well, the tone of the bladder having been quite recovered.

I remember some years ago seeing a case which had been under the care of a surgeon, since deceased, who had declared that the patient was suffering from malignant disease of the colon, and as the gentleman was eighty-four years of age he was advised that operation would be inadvisable. I found a curiously oblong tumour passing obliquely upwards to the right side, and reaching nearly to the liver.. Tracing it downwards it passed into the pelvis in the central line. It was dull on percussion, but 
not solid. I found that he had a very large prostate, and on asking if he had difficulty in passing urine I elicited all the usual symptoms. A catheter solved the difficulty, for the tumour promptly disappeared. I mention this case in connection with the other in order to show that even an experienced surgeon may be misled by a distended bladder, and that the possibility of such a thing should never be forgotten when dealing with tumours of the abdomen.

Dangers of Operation.-What of the dangers of the operation? Apart from the fact that we are dealing with aged persons, who are often broken down by suffering of the most distressing character, there are undoubtedly risks. of a serious kind. We make an opening into the bladder suprapubically, and the escaping urine, in spite of drainage tubes or siphonage, does to some extent come into contact with the wound. If the urine is from a bladder which has been severely inflamed, and is putrid, the dangers are increased. It is of importance to limit the risks as much as possible. In the first place, I always secure the cut edges of the bladder wound to the fibres of the separated rectus muscle. Besides lessening the chances of extravasation it has the advantage that when changing the drainage tube the sutures on either side serve as a guide in its re-introduction, and we are spared the risk of breaking down adhesions by trying to push the tube through an opening which in the early stages rapidly narrows.

Then the surgeon is often alarmed by the perilously. close approximation of the finger in the bladder to the finger in the rectum. In a thin bladder it sometimes feels as if the least additional effort would make the fingers actually meet. The accident has happened in more than one case; but let me say in passing that the perineal operation cannot claim immunity in this respect, for several cases of recto-vesical fistulæ have been reported 
as a result of that procedure. I refer to this matter simply to emphasise the lesson that while undoubtedly we must use much force in some cases where the connecting fibres are very tough, our violence must be gentle, if I may so say, and, above all things, under control. There must be no rough pulling upon bands, but a careful peeling off of all attachments, with the finger nail always directed close to the surface of the prostate.

In some cases the peritoneal fold falls freely into the upper part of the wound, and ought to be carefully protected. This may be done by a retractor of the Simon type, or it may be tucked up under a pad of sterile gauze and given in charge of an assistant.

The Trendelenburg position is of use if a close inspection of the interior of the bladder is required, but I never xuse it for getting the peritoneal fold out of the way. As is now admitted, the position is attended by risks, and in the case of the old patients with whom we have to deal it is especially dangerous to crowd the viscera against the diaphragm and so interfere with the action of the lungs and heart.

Primary Hamorrhage.-As to free primary hæmorrhage, I have only encountered it once, and I have already reported that case. I have for some time adopted the plan of administering ten-grain doses of chloride of calcium three times a day for one and a half or two days before operation. The result has been entirely satisfactory, and the bleeding during the actual enucleation has been very small.

Drainage.-At the close, water as hot as can be borne by the finger is used to douche the bladder until it returns quite clear, then the disturbed membrane in the position of the prostate is gently replaced, and the tube is secured in the wound. A rectangular glass tube, to one arm of 
which a rubber tube has been fixed, is passed into the bladder, and to the other arm, which lies on the abdomen, supported by a layer of wadding, a long rubber pipe is attached and passes into a bottle holding a known quantity of water, the level of which is marked. Any rise above this point shows whether urine is flowing, but this can always be seen by looking at a glass joint fixed midway in the length. This plan has been used by Sir Thornley Stoker, and I have found it very serviceable in the first three or four days during which the tube is retained in the bladder.

Fatal Cases.-The mortality may be thus summed up :One died of ether bronchitis-he was aged seventy-five, and suffered from mitral disease; one died fourteen days after operation from a suddenly-developed peritonitis; one from pelvic cellulitis; one died in twenty-four hours from sepsis; and one died twelve weeks after operation from gangrene of the right lung. This case was sufficiently well to be out of bed for a couple of weeks, and the wound was nearly closed, when he had an attack of purpura hæmorrhagica. He wasted considerably, and all progress ceased. He recovered from this, but four weeks later he had another attack of purpura. Blood came from the bladder, lungs, nose, and intestine. He developed cough and much bloody expectoration. At the postmortem the right lung was in a state of gangrene. This death can hardly be attributed to the operation, and if I omit it I have lost only one in the last ten.

Cases for Operation.-I may say, however, that I have never refused to operate in any case, no matter how feeble or advanced in years, when the question to be dealt with was the attempt to relieve the patient from intolerable distress. I have treated many by means which are generally accepted as less heroic and so often capable of giving 
a large degree of comfort. It would be very foolish to lay down the law that all cases of enlarged prostate are to be operated upon. Many men have enlarged prostates, but certainly not all enlarged prostates demand removal. But I have no doubt that if the nocturnal distress is great, if the patient has had recurring attacks of retention, if he has suffered from outbreaks of cystitis, if he has had severe hæmorrhage, if he has suffered from any one or all of these, then operation ought to be undertaken. The risks attending these troubles are in a sense more certain than the risks of enucleation. The gratitude of a patient who bas been restored to the ordinary method of urination, whose sleepless nights and frequent painful efforts to empty the bladder have gone for ever, impels one who has to do with such cases to urge the advantage of operation. The patients seem to rejuvenate, and are enabled to live a life of peace, at least in this respect.

Sir Thornley Stoker expressed himself in doubt as to the accuracy of statistics concerning the mortality resulting from operation for prostatic enlargement. The time required for prostatic enucleation, in his experience, varied. He had enucleated a large prostatic growth in two minutes, but in another case in which the growth was small the enucleation took half an hour. He recommended benzoate of sodium as a useful drug. for promoting acidity of the urine. He considered the Trendelenburg position attended by danger in the case of aged patients.

Sir ARThur Chance favoured operative measures directed towards the relief of patients suffering from the effects of senile prostatic enlargement. He had found large prostates easy to deal with, and he had frequently noticed that small prostates. gave the most trouble. In fatal cases the cause of death was. usually intra-thoracic complications. He regarded the Trendelenburg position of great assistance, and he had not found it attended by any obvious risk. 
SrR William Thomson, in replying, stated that there was no direct relationship between the degree of prostatic enlargement and the intensity of the symptoms. He alluded to cases of death following suddenly after operation, and which were due evidently to embolism. With regard to washing out the bladder, he usually did so through the urethra forty-eight hours after the enucleation. 